\begin{tabular}{|c|l|}
\hline Title & Surface plasmon resonance for detecting clenbuterol: Influence of monolayer structure \\
\hline Author(s) & Suherman; Morita, Kinichi; Kawaguchi, Toshikazu \\
\hline Citation & $\begin{array}{l}\text { A pplied Surface Science, 332, 229-236 } \\
\text { https://doi.org/40.1016/.apsusc.2015.01.169 }\end{array}$ \\
\hline Issue Date & 2015-03-30 \\
\hline Doc URL & http://hdl.handle.net/2115/64909 \\
\hline Rights & $\begin{array}{l}\text { ○ 2015. This manuscript version is made available under the CC-BY-NC-ND 4.0 license } \\
\text { http://creativecommons.org/icenses/by-nc-nd/4.0/ }\end{array}$ \\
\hline Rights(URL) & http://creativecommons.org/icenses/by-nc-nd/4.0/ \\
\hline Type & A pplied Surface Science 332 (2015) 229 236.pdf \\
\hline article (author version)
\end{tabular}

Instructions for use 


\section{Surface Plasmon Resonance for Detecting Clenbuterol: Influence of Monolayer Structure}

Suherman $^{a, b}$, Kinichi Morita ${ }^{c}$, Toshikazu Kawaguchi ${ }^{a, *}$

${ }^{a}$ Division of Environmental Materials Science, Graduate School of Environmental Science and Section of Materials Science, Faculty of Environmental Earth Science, Hokkaido University, Sapporo 060-0810, Japan.

${ }^{b}$ Department of Chemistry, Faculty of Mathematics and Natural Sciences, Universitas Gadjah Mada, Sekip Utara Kotak Pos 21 BLS Yogyakarta 55281, Indonesia.

${ }^{c}$ New Business Development Office, USHIO INC. 6-1 Ohtemachi 2-chome, Chiyoda-ku, Tokyo, 100-8150, Japan

\section{*Corresponding Author}

Email: t_kawa@ees.hokudai.ac.jp

Tel.: +81-11-706-2289

Fax: +81-11-706-2289 


\section{ABSTRACT}

Surface plasmon resonance sensor equipped with a fabricated immunosensor chip is used for detecting clenbuterol in this study. Since clenbuterol is a small analyte, indirect competitive inhibition immunoassay is employed. For fabricating the immunosurface, the Au-chip was functionalized by succinimidyl-terminated alkanethiol, and the terminal $N$-hydroxysuccinimide group of the self-assembled monolayer was either replaced with clenbuterol or blocked with ethanolamine. Scanning tunneling microscope experiments and electrochemical measurements depicted the domain structures of the succinimide group of succinimidyl-terminated propanethiol monolayer. The surface concentration and the orientation of succinimide group was significantly dependent on the concentration of dithiobis(succinimidyl) propionate (DSP) used in fabricating the monolayer. Furthermore, the structure of monolayer significantly influenced both the surface concentration and the orientation of clenbuterol on the sensor surface. Consequently, high coverage and standing-up configuration of clenbuterol showed high affinity for clenbuterol antibody. However, high affinity constant exhibited by the sensor surface was coupled with a low sensitivity. By contrast, lowest concentration of DSP solution (0.1 mM) used in fabricating the immunosurface showed a detection sensitivity of 3 ppt-the highest reported sensitivity for clenbuterol. For regeneration the immunosurface, $0.1 \mathrm{M} \mathrm{NaOH}$ was used and the same sensor surface could be reused for performing >100 rapid immunoreaction.

Keywords: Surface plasmon resonance, self-assembled monolayer, clenbuterol detection, indirect competitive inhibition immunoassay. 


\section{Introduction}

In recent decades, surface plasmon resonance (SPR) sensors have been developed for numerous applications in fields, such as drug discovery, environmental monitoring, food safety, and security [1-6]. SPR is an optical transducer based on surface plasmon phenomena. SPR can measure the dielectric constant changes of the interface caused by the binding of target analytes

with biointerfacial materials. In our SPR system, the sensitivity can be reached up to $30 \mathrm{pg} / \mathrm{cm}^{2}$ (0.3 RU) in case of protein adsorption. In order to add high selectivity, biochemical recognition elements, such as antibodies, enzymes, proteins, DNA and cells are immobilized on the solid surface of the SPR sensor [7-10]. Biochemical recognition elements can be immobilized by physical adsorption [11], by embedding in polymers or membranes [12,13], by trapping in solgels $[14,15]$, and by using functionalized alkanethiol or functionalized alkylsilane self-assembled monolayer (SAM) [16,17]. Each immobilization approach has advantages. For example, the fabrication of protein-conjugate physically adsorbed on the sensor chip (protein-conjugate/sensor chip) is performed in a single step. With a capacity to store large quantities of the biochemical recognition elements, polymer, membrane, and sol-gel/sensor chips show a large signal response to mass change. Although SAM has the disadvantage of low signal response of mass transducer because immobilization on a flat surface limits the number of accessible biorecognition elements, its sensitivity and stability are remarkably high.

The preparation of SAM is straightforward; the immersion of an appropriate substrate into a solution containing a functionalized alkanethiol or a functionalized alkylsilane leads to the spontaneous formation of a mololayer $[18,19]$. The monolayer structure is considered to influence the sensitivity and stability; therefore, researchers direct their efforts to form an ideal monolayer structure that is close-packed and defect-free. However, analyses by the scanning 
probe microscope reveal that monolayers consist of ordered and disordered domains [20-24]. Moreover, pinholes and defects are often observed at domain boundaries; analytes and impurities can be adsorbed onto these vacant sites and the mass change due to nonspecific adsorption can significantly affect the sensitivity and selectivity.

Since SH-group of alkanethiol covalently binds to metal, the domain structure of alkanethiol monolayer partially reflected to the surface structure of underlying substrate, such as terrace, step, plane indices, and crystalline structure. Several academic researchers use a single crystalline metal substrate; however, since polycrystalline metal substrates are used in sensor chips with practical applications, the monolayer structure on polycrystalline metal substrate will be discussed here.

Monolayer formation is very sensitive to the cleanliness of the substrate surface. If the surface is partially covered with contaminants, the surface coverage of SAM is low. Thus, the metal substrate requires sufficient cleaning prior to the fabrication of the SAM. In this study, the surface of the metal substrate is cleaned or ashed by irradiation with an excimer light with oxygen, a method that is widely used for cleaning semiconducting wafers. Ozone produced by exciting oxygen using excimer light $(\lambda=172 \mathrm{~nm})$ oxidizes the contaminants on the metal, thereby cleaning the surface without damaging it.

Here, dithiobis(succinimidyl) propionate (DSP) is used for immobilizing an antigen onto a Au biosensor chip. DSP-a widely used commercially available reagent-has a short alkyl chain, a thiol and a succinimide at its terminal. Thiol terminal of the DSP binds to the Au surface and a biochemical recognition element with an $\mathrm{NH}_{2}$-terminal can be instantly immobilized, since succinimide group of DSP is replaced by amines in a neutral buffer in a single step. However, 
since alkanethiols with short alkyl chains are often shown to adopt a lying-down phase [25,26], the orientation of biochemical recognition element can significantly affect the sensitivity of the biosensor. We will, therefore, discuss the relationship between the sensitivity of the sensor and the lying-down configuration of the molecules in domain structure.

The analyte chosen for this study is clenbuterol, an illegal ingredient in meat from food producing animals. Conventional analyses of clenbuterol including chromatography method, ELISA, electrophoresis, and electrochemistry involve complex time-consuming steps (such as incubation and repeated washings) can take up to $72 \mathrm{~h}$ for a single sample, with sensitivity detection at ppb levels [27-32]; thus, there is an urgent need for a highly sensitive sensor to detect clenbuterol to ensure food safety. However, the detection of small molecule clenbuterol $(\mathrm{Mw}=277)$ by a mass transducer using the direct immunoassay is challenging. In this study, we employ the indirect competitive inhibition immunoassay for detecting the small analyte [6, 3336]. This method ensures that the signal response of the mass transducer is large, because the antibody, rather than the antigen, is being measured. Thus, reveals relatively higher sensitivity at ppt level. Results from previous reports [10,17] predict that the orientation of immobilized antigen on the sensor surface influences the sensitivity of the detection. Therefore, this study will also discuss the molecular scale structure in the context of sensing performance.

\section{Experimental}

\section{Materials}

Potassium hydroxide and ethanol were obtained from WAKO, Japan, while Sodium hydroxide was from Junsei chemical Co., Ltd., Japan. DSP and phosphate buffer saline (PBS) were purchased from Sigma Aldrich, USA. Methanol was obtained from Dojindo, Japan. 
Clenbuterol hydrochloride and monoclonal mouse IgG antibody of clenbuterol (Ab) were ordered from LKT laboratories, Inc., USA and Novus Biologicals, USA, respectively. Refractive index matching fluid (refractive index $=1.518$ ) was obtained from Cargille Labs., USA. All chemicals were of reagent or higher grade, and water $(18.2 \mathrm{M} \Omega \mathrm{cm})$ from a Millipore system was used in all experiments.

\section{Preparation of Au substrates}

Glass substrates (BK7 type, $20 \mathrm{~mm}$ x $13 \mathrm{~mm}$ x $0.7 \mathrm{~mm}^{\mathrm{t}}$ from Matsunami Glass Ind., Ltd., Japan) were sonicated in soap water (10\% Contrad 70 detergent from Fisher). After rinsing with sufficient water, the glass substrates were dried with pure-nitrogen gas. In order to make the surface hydrophilic, the glass substrates were cleaned using plasma at $15 \mathrm{~W}$ under 2.0-2.5 Pa. Subsequently Au (50 nm) was sputtered onto the glass chips under 2.0-2.5 Pa. The prepared Auchips were loaded into the SPR sensing system immediately after excimer ashing pretreatment (172 nm of incident light) for 2 min.

Reported surface densities of monolayers were corrected for the surface roughness of Auchip $(\delta=1.50)$, which was calculated from the reduction charge density of Au-O in the cyclic voltammogram in $0.1 \mathrm{M}$ sulfuric acid.

\section{Electrochemistry}

Electrochemical measurements were performed in an electrochemical cell holding using HZ-5000 Automatic Polarization System (Hokuto Denko, Japan). Experiments were conducted with a conventional three-electrode system with the Au-chip substrate, a platinum wire, and $\mathrm{Ag} / \mathrm{AgCl}$ (saturated $\mathrm{KCl}$ ) as the working electrode, the counter electrode and the reference 
electrode, respectively. Aqueous potassium hydroxide (0.1 M), deaerated with $5 \mathrm{~N}$ Ar in a glove box, was used as the electrolyte solution.

\section{Scanning tunneling microscopy (STM)}

STM experiments were conducted using a NanoScope STM III (Digital Instruments, USA) operating in the constant current mode (a few hundred picoampere) with the bias voltage typically between 0.5 and $1 \mathrm{~V}$. For imaging, Pt/Ir (80/20) mechanically cut tips (diameter $=0.25$ $\mathrm{mm}$, Bruker, USA) were used to scan the surface.

\section{X-ray photoelectron spectroscopy (XPS)}

XPS experiments were conducted using a Rigakudenki model XPS-7000 X-ray photoelectron spectrometer. Monochromatic Mg Ka radiation is operated at $300 \mathrm{~W}$. The angle of take-off is $90^{\circ}$. For determining the binding energy of elements, Au4f7/2 emission is used as an internal reference.

\section{Surface plasmon resonance (SPR) sensing}

SPR experiments were performed on SPR-670 (Nippon Laser Electronics, Japan) equipped with a fully automated flow system consisting of a plunger pump and an injector. The Au-chip was mounted on the semi-cylindrical prism with a refractive index matching liquid. Red light (670 nm) emitted from Ni-Cd laser was reflected at the Au-coated glass plate at attenuated total reflection angles, and the reflected light intensity was recorded using CCD camera. The reflectance angle, at which the light intensity was minimum (SPR angle), was recorded with time. All the experiments were conducted in an air-conditioned room $\left(25^{\circ} \mathrm{C}\right)$. 


\section{Fabrication of the sensor surface}

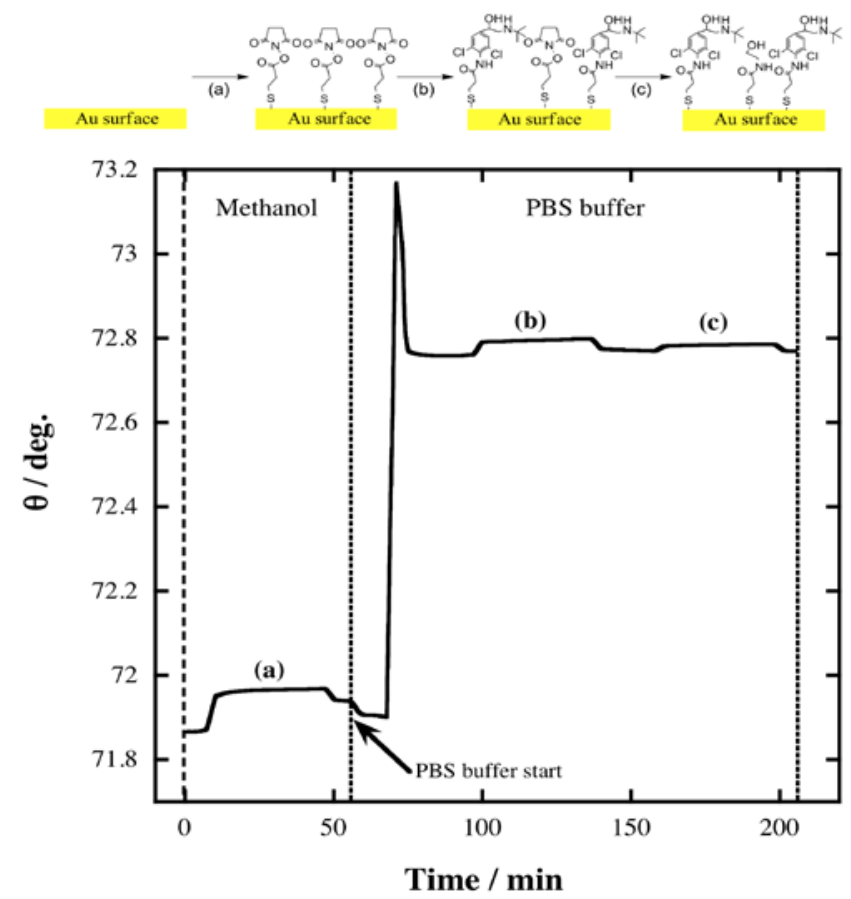

Fig. 1. SPR sensorgram for fabricating the sensor surface: (a) self-assembly of succinimidylterminated propanethiol monolayer, (b) immobilization of clenbuterol by amide coupling reaction, and (c) blocking unreacted $N$-hydroxysuccinimide with ethanolamine. Flow rate was 5 $\mu \mathrm{L} \min ^{-1}$.

Fig. 1 shows the SPR sensorgram of the fabrication process. Initially, methanol was allowed to flow (5 $\mu \mathrm{L} \mathrm{m^{-1 }}{ }^{\text {) }}$ over the surface of unmodified Au-chip until the resonance angle remained stable. Then, a methanolic solution of DSP was injected over a 40-min period (Fig. 1(a)). The Au-chip was covered with the succinimidyl-terminated propanethiol monolayer during this first operation. Subsequently, the running solution was switched from methanol to PBS solution (pH 7.4). Since the refractive indices of methanol and PBS solution are different, there was an abrupt shift in the resonance angle. After stabilizing the resonance angle, a solution of clenbuterol (200 $\mu \mathrm{g} \mathrm{mL}^{-1}$ ) in PBS was injected over a 40-min period (Fig. 1(b)). During this process, the succinimidyl group was replaced by clenbuterol. Even in the presence of excess clenbuterol, unreacted $N$-hydroxysuccinimide ester groups were retained on the Au-chip. These 
$N$-hydroxysuccinimide groups were replaced by ethanolamine (a PBS solution containing ethanolamine $1 \mathrm{mg} \mathrm{mL}^{-1}$ ), i.e., blocking agent, was injected over a 40-min period (Fig. 1(c)). Finally, the sensor surface was used for detecting clenbuterol.

\section{Immunoassays}

An indirect competitive inhibition immunoassay protocol, similar to that reported previously [6,37-39], was employed for detecting clenbuterol. In this work, a solution of clenbuterol antibody (Ab), i.e., a PBS solution containing monoclonal mouse IgG antibody (100 $\mu \mathrm{g} \mathrm{mL} \mathrm{L}^{-1}$ ) of clenbuterol was premixed with the solution of the analyte sample (PBS solution containing clenbuterol) before being injected into the sensing system. As the solution of clenbuterol and its antibody flowed over the sensor surface, SPR sensed the dielectric constant change at the interface due to the binding of the unreacted $\mathrm{Ab}$ to clenbuterol immobilized on the sensor surface. The total shift in the resonance angle was determined from the difference in angle before and after the injection.

\section{Results and discussion}

\section{Characterization of self-assembled monolayer}

Dependence of the monolayer structure of succinimidyl-terminated propanethiol on the concentration of DSP is examined. Fig. 2(a) shows the SPR sensorgram for the self-assembly of the succinimidyl-terminated propanethiol monolayer on Au-chip. Once a stable baseline is obtained at a flow rate of $5 \mu \mathrm{L} \mathrm{min}{ }^{-1}$, the methanolic solution of DSP is injected over a 40 -min period, following which the running solution is switched back to DSP-free methanol. The initial shift in the resonance angle can be attributed to the difference in the dielectric constant (refractive index) between the interface and the running solution on the inclusion of DSP [40]. 
As the running solution returns to methanol after 40-min, the shift in the resonance angle decreases (except for DSP concentration of $0.1 \mathrm{mM}$ ). Thus, the overall shift in the resonance angle $(\Delta \theta)$ is determined as the difference in resonance angles at $t=0 \mathrm{~s}$ and $t=2700 \mathrm{~s}$ (at both instances DSP-free methanol is flowing over the chip) to eliminate the influence of the refractive index of solution. The results indicate that the value of $\Delta \theta$ shows a DSP concentration dependent increase. Since there is no contribution from the refractive index of the solution on the value of $\Delta \theta$, this value directly indicates the dielectric constant of the monolayer. In other words, while the surface concentration of monolayer cannot be estimated directly from $\Delta \theta$, the shift in the resonance angle can show the dielectric constant difference of the monolayer.
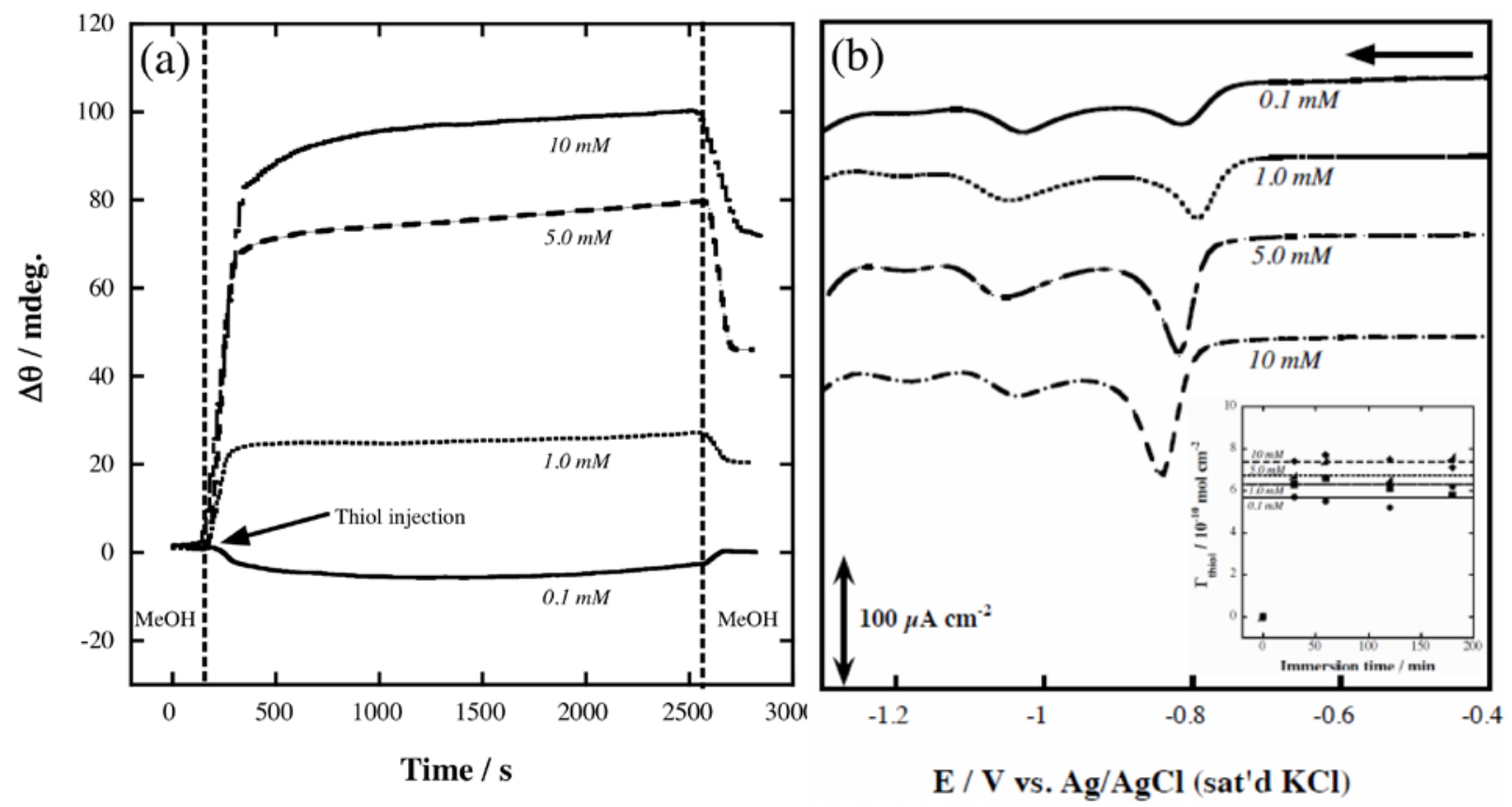

Fig. 2. (a) SPR sensorgrams for the self-assembly of succinimidyl-terminated propanethiol on Au surface prepared in methanolic solution flowing $\left(5 \mu \mathrm{L} \mathrm{min}{ }^{-1}\right)$ on the top of Au surface; (b) voltammograms of reductive desorption of succinimidyl-terminated propanethiol monolayer, electrolyte solution was $0.1 \mathrm{M} \mathrm{KOH}$. Sweep rate was $0.1 \mathrm{~V} \mathrm{~s}^{-1}$. Inset: immersion time dependence on the surface concentrations of succinimidyl-terminated propanethiol monolayer were calculated by reductive desorption. Labeled values are the solution concentration of DSP. 
Electrochemical reductive desorption is performed to investigate the structure of monolayer (Fig. 2(b)). Since S-Au bond can be electrochemically cleaved by one-electron reaction [41], the surface concentration of the monolayer can be estimated from the charge density, which is calculated from the integration of reduction current. The reduction current shows three peaks at approximately $-0.8 \mathrm{~V}$ (from $-0.8 \mathrm{~V}$ to $-0.85 \mathrm{~V}$ ), $-1.04 \mathrm{~V}$ and $-1.19 \mathrm{~V}$, characteristic of reductive desorption on a polycrystalline Au electrode [42]. By comparing the peak positions in the voltammograms of similar systems reported previously, it is considered that the major peak (at approximately $-0.80 \mathrm{~V}$ ) resulted from desorption of alkanethiols from the terraces [40], and the peaks at $-1.04 \mathrm{~V}$ and $-1.19 \mathrm{~V}$ were due to the desorption of alkanethiols adsorbed on the steps [42-45] of polycrystalline Au. The intensities of these reduction peaks increase with an increase in either the immersion time or the concentration of DSP (the width of the major peak shrinks). These results indicate that the surface concentration of monolayer increases with increase in the concentration of DSP. The surface concentration of DSP is in the range from $5.1 \times 10^{-10} \mathrm{~mol} \mathrm{~cm}^{-2}$ to $7.7 \times 10^{-10} \mathrm{~mol} \mathrm{~cm}^{-2}$ (Fig. 2(b)-Inset). With a 30-min immersion time, the surface concentration reaches saturation, a result consistent with the results from SPR studies. From XPS analysis (Table S1, Supporting Information), Au4f signal decreased with increasing DSP solution concentration; implies that the photoelectron of Au4f was interrupted by the thiol monolayer. Thus, it said that the thickness and the surface concentration of monolayer covered on Au increased with increasing DSP solution concentration. In addition, tendency of S2p/Au4f was almost corresponding to the surface concentration estimated by reductive desorption charge density. However, this ratio is dependent on both thickness and surface concentration. Thus, it could not be translated to the thickness of monolayer, directly. 
According to the Langmuir adsorption isotherm (data taken from Fig. 2(b)-Inset), the Langmuir equilibrium constant $(K)$ and the maximum surface concentration $\left(x^{\infty}\right)$ of succinimidyl-terminated propanethiol onto Au-surface are estimated to be $1.14 \times 10^{4} \mathrm{M}^{-1} \mathrm{~cm}^{2}$ and $7.710^{-10} \mathrm{~mol} \mathrm{~cm}{ }^{-2}$. The equilibrium constant $K$ for binding succinimidyl-terminated propanethiol to Au is comparable to that of octadecanethiol $\left(1.08 \times 10^{4} \mathrm{M}^{-1} \mathrm{~cm}^{2}\right.$ to $1.53 \times 10^{4} \mathrm{M}^{-}$ $\left.{ }^{1} \mathrm{~cm}^{2}\right)$ and larger than that of octanethiol $\left(1.9 \times 10^{3} \mathrm{M}^{-1} \mathrm{~cm}^{2}\right)$ [46,47], suggesting that the strength of the molecular cohesion of succinimidyl-terminated propanethiol is equal to the strength of van der Waals force interactions between long alkyl chains. However, the value of $\mathrm{x}^{\infty}$ for succinimidyl-terminated propanethiol is lesser than that of alkanethiol monolayer $\left(1.1 \times 10^{-9} \mathrm{~mol}\right.$ $\mathrm{cm}^{-2}$ ) [48] because the area occupied by succinimide group is larger than that of methyl group. In addition, the precise surface concentration of DSP could not be determined in this experiment because the local minimal value (potential of zero charge) is not observed in the differential capacitance curve in potassium hydroxide (0.1 M; data not shown). Therefore, the surface concentration is measured with approximately $30 \%$ error [48].

The peak potential of the reductive desorption also provides insights into the monolayer structure. The potential of the major peak is shifted to more negative potential with increase in the concentration of DSP in the range from $1.0 \mathrm{mM}$ to $10 \mathrm{mM}$. However, there is a discernible shift in the peak potential of the major peak towards more negative values when the concentration of DSP is reduced from $1.0 \mathrm{mM}$ to $0.1 \mathrm{mM}$. The peak potential is indicative of the permeability of the solvent into the monolayer [49]. When the monolayer is prepared in solution, where the concentration of DSP $>1.0 \mathrm{mM}$, it is considered that the density of each domain becomes condensed, and the domain size increases with the increase in the concentration of DSP. However, when the monolayer is prepared in $0.1 \mathrm{mM}$ DSP solution, the solvent hardly penetrates 
inside the monolayer; this indicates that the molecules in the monolayer adopt a lying-down configuration and cover the propanethiol surface.
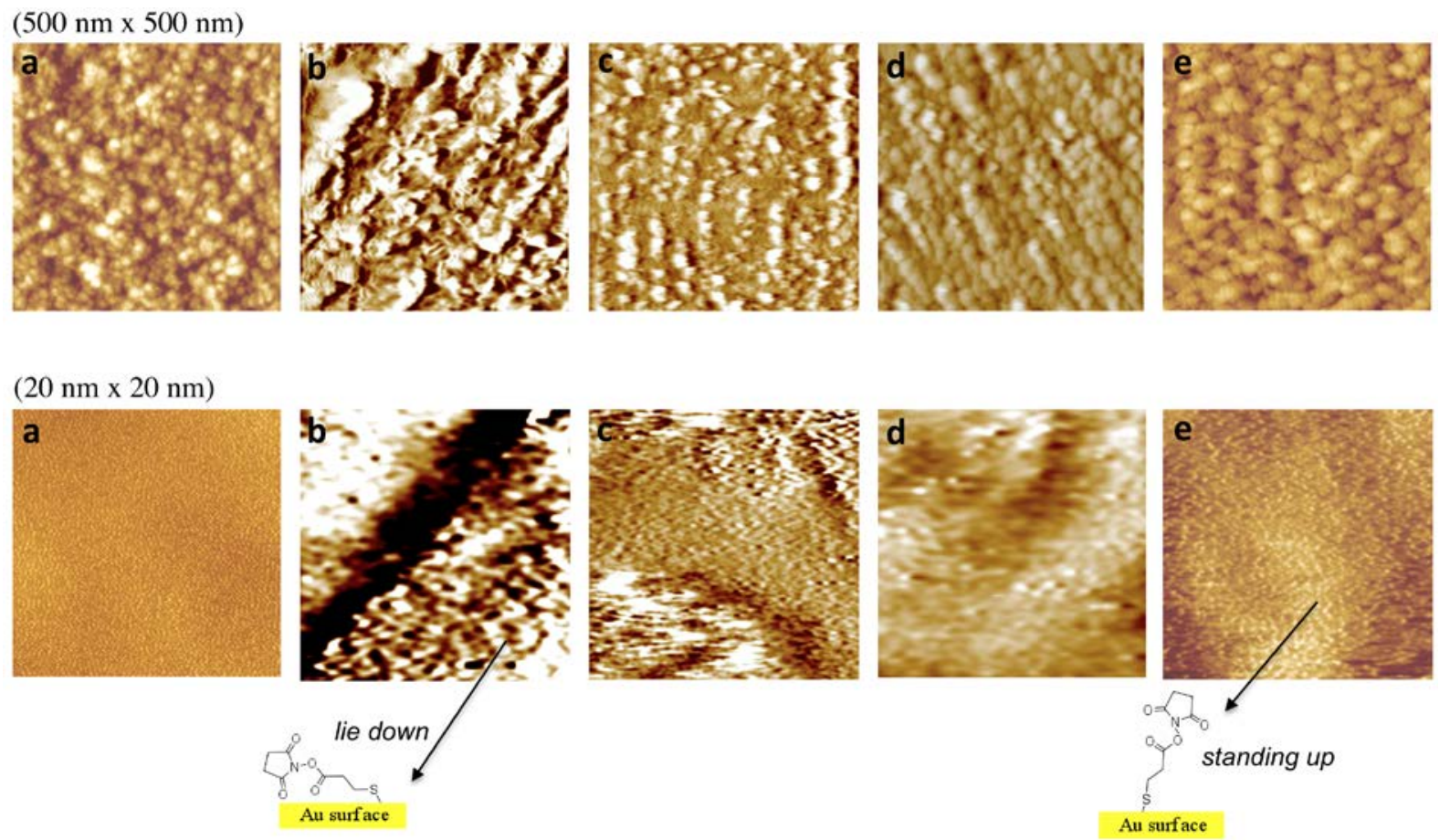

Fig. 3. STM images of the Au-chip (a) before and after modification by immersion in a solution of dithiobis(succinimidyl) propionate (DSP) with various concentrations, (b) $0.1 \mathrm{mM}$, (c) 1.0 $\mathrm{mM}$, (d) $5.0 \mathrm{mM}$, and (e) $10 \mathrm{mM}$. The bottom panel shows a high-resolution image of the surface shown in the top panel.

Fig. 3 and Fig. 4 show the STM images of the succinimidyl-terminated propanethiol monolayer and the fabricated sensor surface containing clenbuterol and ethanolamine-terminated propanethiol monolayer. In Fig. 3, the top panel shows the images of the different surface at lower resolution, while the same surface is magnified in bottom panel. In the STM image of unmodified Au (Fig. 3(a)), coarse grainy structures (approximately $15 \mathrm{~nm}$ ) are observed at higher resolution [50-52]. Since the distance between the grains is about $0.14 \mathrm{~nm}$, it is likely that these grains represent the Au atoms. Low-resolution images of succinimidyl-terminated propanethiol monolayer (Fig. 3(b-e)) do not show any significant differences from that of the unmodified Au. 
A spread of high-density regions on the terraces of Au islands is barely discernible in these images. High-resolution images resolved the white regions in the low-resolution images into separate grains. The monolayer prepared with DSP at $0.1 \mathrm{mM}$ shows small-interconnected grains. The size of interconnected structure was coinciding to succinimide group (ca. $0.40 \mathrm{~nm}$ in diameter). The interconnected structures in the high-resolution image (Figure 3b, bottom panel) can be formed because of lying-down configuration of the succinimidyl-terminated propanethiol monolayer. With increase in the concentration of DSP, the regions of lying-down configuration became smaller, while domains with a grainy and coarse structure continue to grow till the surface is almost full covered with the grains (at $10 \mathrm{mM}$ DSP). These observations are consistent with the position of the potential of major reductive desorption peak (Fig. 2(b)). When the succinimide groups formed lying-down configuration in the monolayer prepared in $0.1 \mathrm{mM}$ DSP solution, desorption is weak since the solvent does not efficiently penetrate the monolayer. When the monolayer is prepared in $1.0 \mathrm{mM}$ DSP solution, a fraction of the succinimide groups are in standing-up phase: the domain boundaries become clear (Fig. 3(c), lower panel) and the solvent can penetrate such domain boundaries efficiently. At higher concentrations of DSP, domains with succinimide groups in the standing-up phase expand (consequently, domain boundaries between the standing-up and lying-down phase decrease), resulting in reduced penetration of the desorption-facilitating solvent inside the monolayers. 


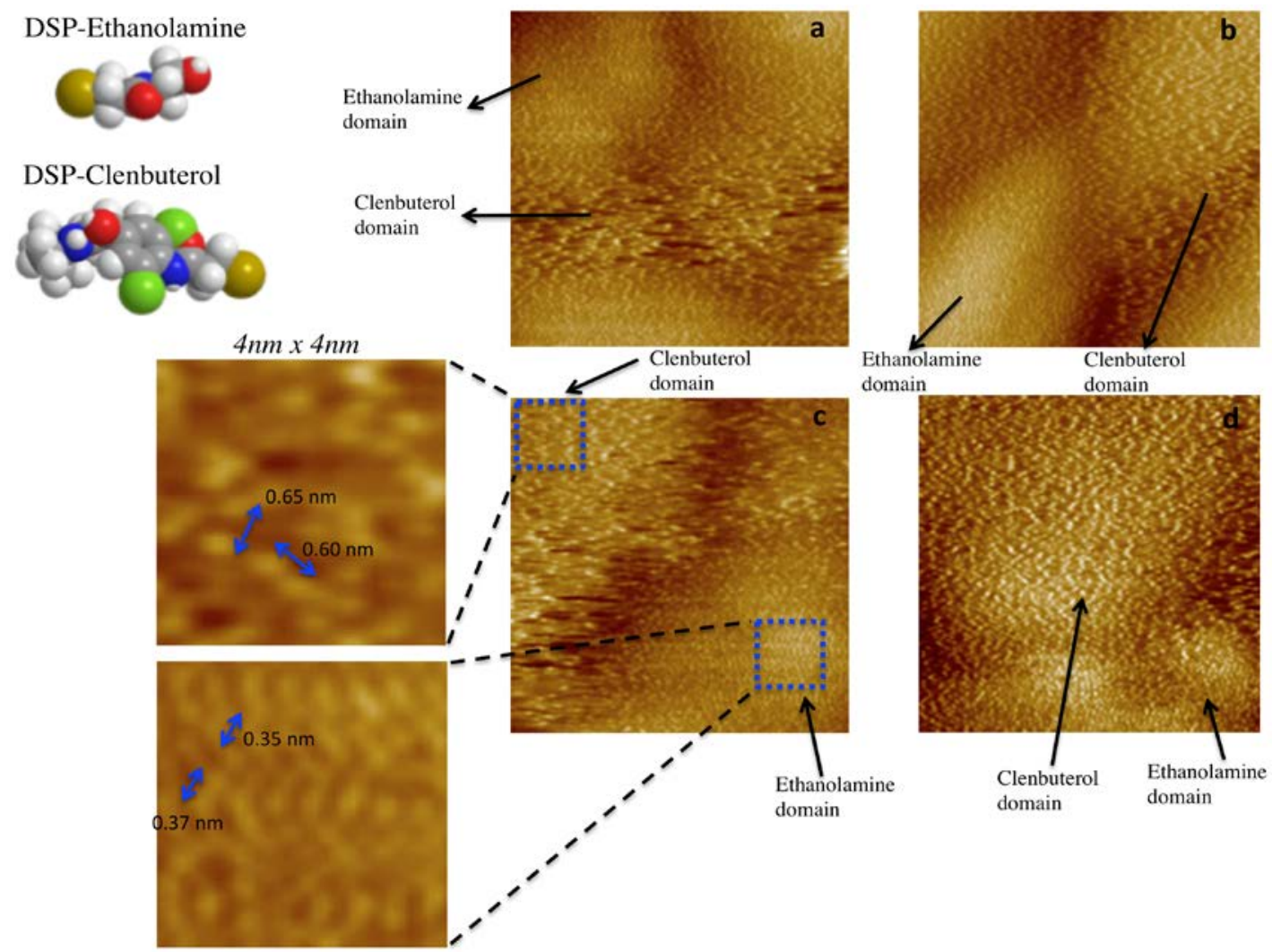

Fig. 4. STM images $(20 \mathrm{~nm} \times 20 \mathrm{~nm})$ of the sensor surface after the immobilization of clenbuterol and ethanolamine. Concentration of dithiobis(succinimidyl) propionate (DSP) used for generating precursor self-assembled monolayer: (a) $0.1 \mathrm{mM}$, (b) $1.0 \mathrm{mM}$, (c) $5.0 \mathrm{mM}$, and (d) $10 \mathrm{mM}$.

STM images of the sensor surfaces modified with clenbuterol- and ethanolamineterminated propanethiol monolayers show that the order of atoms (spots) in each domain is manifested differently (Fig. 4). Considering that the distance between amide $\mathrm{O}$ and alcohol $\mathrm{O}$ in ethanolamine, amide $\mathrm{O}$ and benzyl $\mathrm{O}$ in clenbuterol, and amide $\mathrm{N}$ to secondary alkyl $\mathrm{N}$ in clenbuterol are estimated to $0.40 \mathrm{~nm}, 0.69 \mathrm{~nm}$, and $0.72 \mathrm{~nm}$, respectively (ChemBio3D Ultra Bio 12.0), each molecule (ethanolamine and clenbuterol) can be identified. The increase in coverage by clenbuterol correlates with the increase in the concentration of the DSP used to modify the Au-chips. Although the estimated surface concentrations of both clenbuterol and ethanolamine increase with increase in the concentration of DSP, the percentage of coverage by ethanolamine 
is decreased (Table S2, Supporting Information). In summary, the concentration of DSP determines the surface composition of clenbuterol and ethanolamine.

\section{Detection of clenbuterol}

Indirect competitive inhibition immunoassay is employed for detecting clenbuterol. SPR senses the change in dielectric constant due to the binding of antibody by the immobilized antigen (clenbuterol) at the interface. A large signal change is typically expected in this assay; however, unraveling the kinetic aspects of this assay [34,53-56] can be challenging because the $\mathrm{Ab}$ competitively binds either to immobilized antigen or antigen in the sample solution. Moreover, several kinetics studies do not consider the two immunoreactions, i.e., during premixing and sensing. Here, we have made an attempt to discuss the kinetics in more detail.

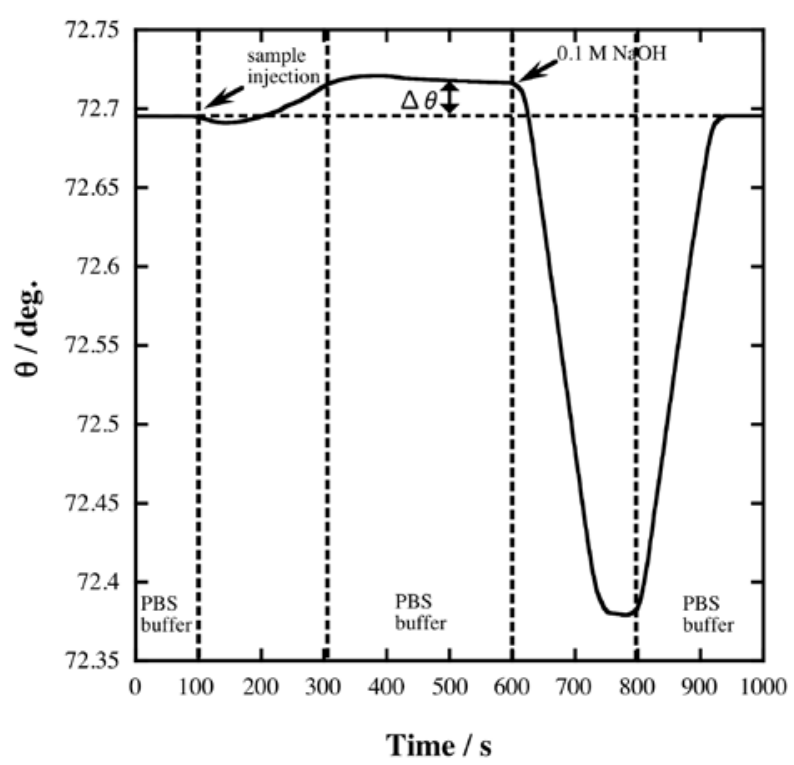

Fig. 5. SPR sensorgram for the immunoreaction of immobilized clenbuterol with Ab standard solution. PBS (10\% ethanol) solution is used as the running solution (100 $\left.\mu \mathrm{L} \mathrm{min}{ }^{-1}\right)$, and $0.1 \mathrm{M}$ $\mathrm{NaOH}$ aqueous solution is used for regenerating the sensor surface. 
Fig. 5 shows the representative sensorgram of immunoreaction with Ab. While Ab standard solution flows over the sensor surface for $200 \mathrm{~s}$ (from $t=100 \mathrm{~s}$ to $t=300 \mathrm{~s}$ ), the resonance angle shifts gradually to higher values with the binding of Ab by the immobilized antigen onto the sensor surface. Difference in the resonance angle before $(t=100 \mathrm{~s})$ and after $(t=$ $400 \mathrm{~s})$ the binding of $\mathrm{Ab}$ to the immobilized antigen is the total resonance angle shift $(\Delta \theta)$ of the immunoreaction: this value is used for the concentration dependence curves.

After the immunoreaction, the sensor surface is regenerated by a solution of sodium hydroxide $(0.1 \mathrm{M})$ till the value of the resonance angle returns to the initial value, indicating that the $\mathrm{Ab}$ is detached from the sensor surface. Through such a regeneration process, the sensor surface can be reused for over 100 immunoreactions. One immunoreaction-regeneration cycle takes only $1000 \mathrm{~s}$. Therefore, the same sensor chip is used for evaluating the immunoreaction in various solutions to determine the kinetic parameters. Moreover, $<5 \%$ error is observed between the results obtained using different sensor chips $(\mathrm{N}>3)$.
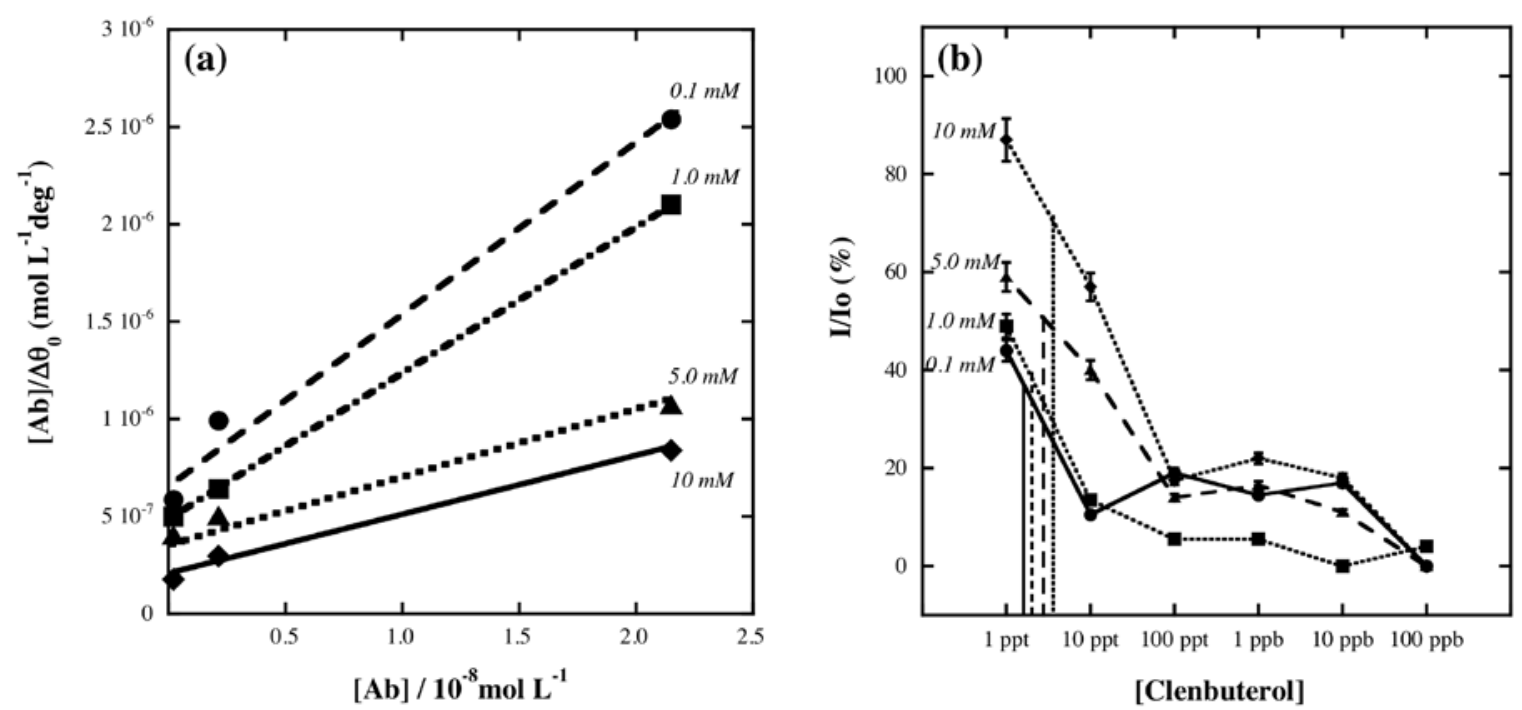

Fig. 6. (a) Langmuir adsorption isotherm plot for binding clenbuterol antibody (Ab) to clenbuterol immobilized on the sensor surface, and (b) clenbuterol solution concentration dependence by using indirect competitive inhibition immunoassay. Inhibition rate was calculated from the angle shift in presence $(\mathrm{I}) /$ absence $\left(\mathrm{I}_{0}\right)$ of clenbuterol. In the premixing process, $1 \mathrm{ppm}$ 
Ab was mixed with series of clenbuterol concentrations. The incubation time was $2 \mathrm{~min}$. Labeled values are the concentration of dithiobis(succinimidyl) propionate (DSP) used in fabricating the sensor surface.

According to reported protocols [34,53-56], the affinity constant $\left(K_{1}\right)$ of Ab to the clenbuterol (antigen) immobilized on the sensor surface can be determined using a Langmuirtype adsorption model (Fig. 6(a)). The Langmuir adsorption isotherm equation for immunoreaction between $\mathrm{Ab}$ and the immobilized clenbuterol on the sensor surface (Ss) is given by:

$$
\begin{gathered}
A b+S s \rightleftharpoons A b-S s \\
K_{1}=\frac{[A b-S s]}{[A b][S s]}
\end{gathered}
$$

Fundamental equation of Langmuir-type adsorption model is expressed as follows:

$$
\frac{[A b]}{\Delta \theta_{0}}=\frac{[A b]}{\Delta \theta_{0, \max }}+\frac{1}{K_{1} \Delta \theta_{0, \max }}
$$

where $[\mathrm{Ab}]$ is concentration of the clenbuterol antibody. $\Delta \theta_{0}$ and $\Delta \theta_{0, \max }$ are the total angle shift for a given concentration of $\mathrm{Ab}$ standard solution and the maximum angle shift with saturated $\mathrm{Ab}$ on the sensor surface, respectively. $\Delta \theta_{0, \max }$ and $K_{1}$ are determined from the slope and the intercept of the Ab standard solution concentration dependence as shown in Fig. 6(a). These parameters are summarized in Table 1.

$K_{1}$ is observed to increase with increase in the concentration of DSP used in fabricating the sensor surface. This result can be rationalized considering $\Delta \theta_{0, \max }$ and the surface composition estimated from STM images (Fig. 4). Coverage by clenbuterol is approximately $40 \%$ when the 
Au chip is modified with a $0.1 \mathrm{mM}$ DSP solution, where it is $>70 \%$ for $10 \mathrm{mM}$ DSP. As expected, the reactivity of the sensor surface is proportional to the surface coverage of reactive site (antigen).

Fig. 6(b) illustrates the clenbuterol concentration dependence by using indirect competitive inhibition immunoassay. During premixing, the following reaction occurs:

$$
A b+C l b \rightleftharpoons A b-C l b
$$

where $\mathrm{Clb}$ is clenbuterol in solution. The affinity constant $\left(K_{2}\right)$ of this immunoreaction is given by:

$$
K_{2}=\frac{[A b-C l b]}{[A b][C l b]}
$$

When the sample solution is dispensed onto the sensor surface after the premixing, free-Ab competitively binds to clenbuterol-immobilized sensor surface or clenbuterol (free) in solution, or does not bind at all. Thus, the total concentration of Ab can be defined as follows:

$$
\left[A b_{\text {total }}\right]=[A b-S s]+[A b-C l b]+[A b]
$$

By inserting Eq. 2 and Eq. 5 to Eq. 6, the equation can be rewritten as follows:

$$
\frac{1}{[A b-S s]}=\frac{1}{\left[A b_{\text {total }}\right]}+\frac{1}{\left[A b_{\text {total }}\right] K_{1}[S s]}+\frac{K_{2}}{\left[A b_{\text {total }}\right] K_{1}[S s]}[C l b]
$$

Because SPR senses the dielectric constant change of [Ab-Ss], so [Ab-Ss] can be represented as $\Delta \theta$. Considering the premixing, the solution concentration of clenbuterol after the incubation is 
$[C l b]=[C l b]_{0} e^{-k t}$ and $\alpha=e^{-k t}$, where $k$ is the reaction rate and $t$ is the period of incubation for the premixing. The Eq. 7, when combined with Eq. 3 and considering the premixing, can be rewritten as follows:

$$
\frac{1}{\Delta \theta}=\frac{1}{\Delta \theta_{0}}+\frac{K_{2} \alpha[C l b]_{0}}{\left[A b_{\text {total }}\right] K_{1} \Delta \theta_{0, \max }} \quad \text { with } 0<\alpha<1
$$

Here, $K_{1}$ and $\Delta \theta_{0, \max }$ have already been determined (Eq. 3, Fig. 6(a)). $\alpha$ can be estimated from the incubation time dependence, and $K_{2}$ with constant $\alpha$ is calculated from the slope of Eq. 8 (Table 1). Note that $K_{1}$ is higher than $K_{2}$, irrespective of the concentration of DSP used in fabricating the sensor surface. In other words, Ab prefers to bind to the clenbuterol immobilized on the sensor surface rather than the free clenbuterol in the sample solution.

Table 1. Kinetic parameters for detecting clenbuterol by indirect competitive inhibition immunoassay.

\begin{tabular}{|c|c|c|c|c|}
\hline$[\mathrm{DSP}] / \mathrm{mM}$ & $\begin{array}{l}\Delta \theta_{0, \max } \\
\text { (mdeg.) }\end{array}$ & $K_{1}\left(\mathrm{x} 10^{8} \mathrm{M}^{-1}\right)$ & $\begin{array}{c}r \text { (correlation } \\
\text { coefficient) }\end{array}$ & $\alpha \cdot K_{2}\left(\mathrm{x} 10^{8} \mathrm{M}^{-1}\right)$ \\
\hline 0.1 & 10.5 & 2.19 & 0.9933 & \\
\hline 1.0 & 11.9 & 2.72 & 0.9999 & $0.65 \pm 0.35$ \\
\hline 5.0 & 23.1 & 2.91 & 0.9985 & \\
\hline 10 & 27.9 & 4.42 & 0.9962 & \\
\hline
\end{tabular}

Furthermore, a high sensitivity of the detection is obtained from a low $K_{1}$. With the sensor fabricated in the solution of DSP at $0.1 \mathrm{mM}$, the limit of detection (LOD, the value was extracted from $85 \%$ of residual activity in the sigmoid curve of indirect competitive inhibition method, equal to $15 \%$ of inhibition [57-58]) is approximately $3 \mathrm{ppt}\left(3 \mathrm{pg} \mathrm{mL}^{-1}\right)$. These results illustrate that $\mathrm{Ab}$ easily binds to the antigen (clenbuterol immobilized on the sensor surface) even at a low surface coverage. 
Considering that the size of $\mathrm{Ab}(14 \mathrm{~nm})$ is comparable to the domain size of clenbuterol (10$20 \mathrm{~nm}$ diameter; Fig. 4) on the surface of the sensor, the possibility that Ab is attracted to the clenbuterol domain on the sensor surface can be envisioned. Thus, we presume that the orientation of clenbuterol also plays a significant role in determining the affinity constant $K_{1}$. This result implies that the clenbuterol antibody recognizes the 2-tert-butyl aminoethanol moiety of clenbuterol. The mechanism of recognition of the antigen by the antibody is intriguing, and studies investigating this are underway.

\section{Conclusion}

This study investigated the relationship between the antigen immobilized monolayer structure and the sensing performance of SPR. Since our target was clenbuterol, a small analyte $(\mathrm{Mw}=277)$, the indirect competitive inhibition immunoassay was employed for its detection. To immobilize clenbuterol onto the sensor surface, succinimidyl-terminated propanethiol monolayer was fabricated on a Au-chip by self-assembly. It was found that the orientation of succinimide group was highly dependent on the concentration of DSP used in self-assembly. The $N$ hydroxysuccinimide group of the succinimidyl-terminated propanethiol was replaced with covalently linked clenbuterol at neutral $\mathrm{pH}$. The structure of the succinimidyl-terminated propanethiol monolayer affected the surface concentration and the orientation of clenbuterol on the fabricated sensor surface. The sensor surface prepared modified in a high concentration of DSP solution showed a high affinity for clenbuterol antibody. However, the high affinity constant $K_{1}$, exhibited by the sensor surface was coupled to a low sensitivity. In contrast, the immunosensor fabricated in the lowest concentration of DSP $(0.1 \mathrm{mM})$ showed a remarkably 
high sensitivity ( $\mathrm{LOD}=3 \mathrm{ppt}$ ). This is the highest sensitivity reported for detecting clenbuterol. Furthermore, this immunosurface can be regenerated by $0.1 \mathrm{M}$ sodium hydroxide. One immunoreaction-regeneration cycle took only $1000 \mathrm{~s}$, and the same sensor surface could be used for $>100$ immunoreactions.

\section{Acknowledgement}

Suherman is thankful to the Hitachi Scholarship Foundation for Ph.D financial support and facilitation.

\section{Supporting Information}

Supplementary data associated with this article can be found in the online version at http:

\section{References}

[1] M.A. Cooper, Optical biosensors in drug discovery, Nat. Rev. 1 (2002) 515-528.

[2] J. Homola, Surface plasmon resonance sensors for detection of chemical and biological species, Chem. Rev. 108(2) (2008) 462-493.

[3] M. Farre, E. Martinez, J. Ramon, A. Navarro, J. Radjenovic, E. Mauriz, L. Lechuga, M.P. Marco, D. Barcelo, Part per trillion determination of atrazine in natural water samples by a surface plasmon resonance immunosensor, Anal. Bioanal. Chem. 388 (2007) 207-214. 
[4] X. Lu, H. Zheng, X. Li, X. Yuan, H. Li, L. Deng, H. Zhang, W. Wang, G. Yang, M. Meng, R. Xi, H.Y. Aboul-Enein, Electrochemical DNA biosensor for screening benzene pollutants, Food Chem. 130 (2012) 1061-1065.

[5] M. Petz, Recent applications of surface plasmon resonance biosensors for analyzing residues and contaminants in food, Monatsh Chem. 140 (2009) 953-964.

[6] T. Kawaguchi, D.R. Shankaran, S.J. Kim, K. Matsumoto, K. Toko, N. Miura, Surface plasmon resonance immunosensor using Au nanoparticle for detection of TNT, Sens. Actuators B Chem. 133 (2008) 467-472.

[7] A. Abbas, M.J. Linmas, Q. Cheng, New trends in instrumental design for surface plasmon resonance-based biosensors, Biosens. Bioelectron. 26 (2011) 1815-1824.

[8] X. Fan, I.M. White, S.I. Shopova, H. Zhu, J.D. Suter, Y. Sun, Sensitive optical biosensors for unlabeled targets: A review, Anal. Chim. Acta 620 (2008) 8-26.

[9] M. Piliarik, H. Vaisocherova, J. Homola, Surface plasmon resonance biosensing, Avraham Rasooly and Keith E. Herold (eds.), Method in Molecular Biology: Biosensors and Biodetection, vol. 503 @ Humana Press, a part of Springer Science + Business Media, LLC 2009. DOI 10.1007/978-1-60327-567-5_5.

[10] M. Kyo, K. Usui-Aoki, H. Koga, Label-free detection of proteins in crude cell lysate with antibody arrays by a surface plasmon resonance imaging technique, Anal. Chem. 77 (2005) 7115-7121.

[11] D.R. Shankaran, N. Miura, Trends in interfacial design for surface plasmon resonance based immunoassays, J. Phys. D: Appl. Phys. 40 (2007) 7187-7200. 
[12] A. Bossi, F. Bonini, A.P.F. Turner, S.A. Piletsky, Molecularly imprinted polymers for the recognition of proteins: The state of the art, Biosens. Bioelectron. 22 (2007) $1131-1137$.

[13] K.S. Phillips, J.H. Han, M. Martinez, Z. Wang, D. Carter, Q. Cheng, Nanoscale glassification of gold substrates for surface plasmon resonance analysis of protein toxins with supported lipid membranes, Anal. Chem. 78 (2006) 506-603.

[14] E.H. Lan, B. Dunn, J.I. Zink, Sol-gel encapsulated anti-trinitrotoluene antibodies in immunoassays for TNT, Chem. Mater. 12 (2000) 1874-1878.

[15] R. Liang, J. Qiu, P.A. Cai, Novel amperometric immunosensor based on threedimensional sol-gel network and nanoparticle self-assemble technique, Anal. Chim. Acta 534 (2005) 223-229.

[16] K.V. Gobi, C. Kataoka, N. Miura, Surface plasmon resonance detection of endocrine disruptors using immunoprobes based on self-assembled monolayers, Sens. Actuators B 108 (2005) 784-790.

[17] S.S. Mark, N. Sandhyarani, C. Zhu, C. Campagnolo, C.A. Batt, Dendrimerfunctionalized self-assembled monolayer as a surface plasmon resonance sensor surface, Langmuir 20 (2004) 6808-6817.

[18] J.C. Love, L.A. Estroff, J.K. Kriebel, R.G. Nuzzo, G.M. Whitesides, Self-assembled monolayers of thiolates on metals as a form of nanotechnology, Chem. Rev. 105 (2005) $1103-1169$.

[19] R. Meunier-Prest, G. Legay, S. Raveau, N. Chiffot, E. Finot, Potential-assisted deposition of mixed alkanethiol self-assembled monolayers, Elec. Chim. Acta 55 (2010) $2712-2720$. 
[20] W. Azzam, L. Al-momani, A new striped-phase of decanethiol self-assembled monolayers on $\mathrm{Au}(111)$ formed at a high solution temperature, Appl. Surf. Sci. 266 (2013) 239-244.

[21] A. Riposan, Y. Li, Y.H. Tan, G. Galli, G. Liu, Structural characterization of aldehyde-terminated self-assembled monolayers, J. Phys. Chem. A 111 (2007) 1272712739.

[22] C. Vericat, M.E. Vela, R.C. Salvarezza, Self-assembled monolayers of alkanethiols on Au(111): Surface structure, defects and dynamics, Phys. Chem. Chem. Phys. 7 (2005) $3258-3268$.

[23] G.E. Poirier, Mechanism of formation of Au vacancy islands in alkanethiol monolayers on $\mathrm{Au}(111)$, Langmuir 13 (1997) 2019-2026.

[24] C. O’Dwyer, G. Gay, B. Viaris de Lesegno, J. Weiner, The nature of alkanethiol selfassembled monolayer adsorption on sputtered gold substrates, Langmuir 20 (2004) 81728182.

[25] M.A.D. Millone, H. Hamoudi, L.M. Rodríguez, A. Rubert, G.A. Benitez, M.E. Vela, R.C. Salvarezza, J.E. Gayone, E.A. Sanchez, O. Grizzi, C. Dablemont, V.A. Esaulov, Self-assembly of alkanedithiol on $\mathrm{Au}(111)$ from solution: Effect of chain length and selfassembly conditions, Langmuir 25 (2009) 12945-12953.

[26] V. Chaudhari, H.M.N. Kotresh, S. Srinivasan, V.A. Esaulov, Substitutional selfassembly of alkanethiol and selenol SAMs from a lying-down doubly tethered butanedithiol SAM on gold, J. Phys. Chem. C 115 (2011) 16518-16523. 
[27] L.Y. Zhang, B.Y. Chang, T. Dong, P.L. He, W.J. Yang, Z.Y. Wang, Simultaneous determination of salbutamol, ractopamine, and clenbuterol in animal feeds by SPE and LC-MS, J. Chromatogr. Sci. 47 (2009) 324-328.

[28] G.P. Zhang, X.N. Wang, J.F. Yang, Y.Y. Yang, G.X. Xing, Q.M. Li, D. Zhao, S.J. Chai, J.Q. Guo, Development of an immunochromatographic lateral flow test strip for detection of ß-adrenergic agonist clenbuterol residues, J. Immunol. Methods 312 (2006) 27-33.

[29] S-Y. Sheu, Y-C. Lei, Y-T. Tai, T-H. Chang, T-F. Kuo, Screening of salbutamol residues in swine meat and animal feed by an enzyme immunoassay in Taiwan, Anal. Chim. Acta 654 (2009) 148-153.

[30] J. Pleadin, A. Vulic, N. Persi, D. Milic, N. Vahcic, Ractopamine and clenbuterol urinary residues in pigs as food-producing animals, Food. Technol. Biotechnol. 49 (2011) $517-522$.

[31] S. Sirichai, P. Khanatharana, Rapid analysis of clenbuterol, salbutamol, procaterol, and fenoterol in pharmaceuticals and human urine by capillary electrophoresis, Talanta 76 (2008) 1194-1198.

[32] G. Fan, J. Huang, X. Fan, S. Xie, Z. Zheng, Q. Cheng, P. Wang, Enhanced oxidation and detection of toxic clenbuterol on the surface of acetylene black nanoparticle-modified electrode, J. Mol. Liq. 169 (2012) 102-105.

[33] N. Miura, M. Sasaki, K.V. Gobi, C. Kataoka, Y. Shoyama, Y. Highly selective and sensitive surface plasmon resonance sensor for detection of sub-ppb levels of benzo[a]pyrene by indirect competitive immunoreaction method, Biosens. Bioelectron. 18 (2003) 953-959. 
[34] K. Nagatomo, T. Kawaguchi, N. Miura, K. Toko, K. Matsumoto, Development of a sensitive surface plasmon resonance immunosensor for detection of 2,4dinitrotoluene with a novel oligo (ethylene glycol)-based sensor surface, Talanta 79 (2009) 1142-1148.

[35] D.R. Shankaran, K.V. Gobi, K. Matsumoto, T. Imato, K. Toko, N. Miura, Highly sensitive surface plasmon resonance immunosensor for parts-per-trillion level detection of 2,4,6-trinitrophenol, Sens. Actuators B 100 (2004) 450-454.

[36] D.R. Shankaran, K.V. Gobi, T. Sakai, K. Matsumoto, K. Toko, N. Miura, Surface plasmon resonance immunosensor for highly sensitive detection of 2,4,6-trinitrotoluene, Biosens. Bioelectron. 20 (2005) 1750-1756.

[37] M. Liu, B. Ning, Y. Peng, J. Dong, N. Gao, L. Liu, Z. Gao, Development of indirect determination of ractopamine in pork liver samples based on surface plasmon resonance sensor, Sens. Actuators B Chem. 161 (2012) 124-130.

[38] D.R. Shankaran, K. Matsumoto, K. Toko, N. Miura, Development and comparison of two immunoassays for the detection of 2,4,6-trinitrotoluene (TNT) based on surface plasmon resonance, Sens. Actuators B Chem. 114 (2006) 71-79.

[39] K. Matsumoto, A. Torimaru, S. Ishitobi, T. Sakai, H. Ishikawa, K. Toko, N. Miura, T. Imato, Preparation and characterization of a polyclonal antibody from rabbit for detection of trinitrotoluene by a surface plasmon resonance biosensor, Talanta 68 (2005) 305-311.

[40] K.D. Kihm, S. Cheon, J.S. Park, H.J. Kim, J.S. Lee, I.T. Kim, H.J. Yi, Surface plasmon resonance (SPR) reflectance imaging: Far-field recognition of near-field phenomena, Opt. Lasers Eng. 50 (2012) 64-73. 
[41] M.M. Walczak, D.D. Popenoe, R.S. Deinhammer, B.D. Lamp, C.K. Chung, M.D. Porter, Reductive desorption of alkanethiolate monolayers at gold: A measure of surface coverage, Langmuir 7 (1991) 2687-2693.

[42] K. Shimazu. Y. Hashimoto, T. Kawaguchi, K. Tada, Construction of mixed mercaptopropionic acid/alkanethiol monolayers on polycrystalline gold electrodes using underpotentially deposited lead as the control element, J. Electroanal. Chem. 534 (2002) 163-169.

[43] K. Shimazu. T. Kawaguchi, T. Isomura, Construction of mixed mercaptopropionic acid/alkanethiol monolayers of controlled composition by structural control of a gold substrate with underpotentially deposited lead atoms, J. Am. Chem. Soc. 124 (2002), $652-661$.

[44] C.J. Zhong, J. Zak, M.D. Porter, Voltammetric reductive desorption characteristics of alkanethiolate monolayers at single crystal $\mathrm{Au}(111)$ and (110) electrode surfaces, J. Electroanal. Chem. 421 (1997) 9-13.

[45] M.M. Walczak, C.A. Alves, B.D. Lamp, M.D. Porter, Electrochemical and X-ray photoelectron spectroscopic evidence for differences in the binding sites of alkanethiolate monolayers chemisorbed at gold, J. Electroanal. Chem. 396 (1995) 103-114.

[46] D.S. Karpovich, G.J. Blanchard, Direct measurement of the adsorption kinetics of alkanethiolate self-assembled monolayers on a microcrystalline gold surface, Langmuir 10 (1994) 3315-3322.

[47] N. Camillone, C.E.D. Chidsey, G. Liu, G. Scoles, Superlattice structure at the surface of a monolayer of octadecanethiol self-assembled on Au(111), J. Chem. Phys. 98(4) (1993) 3503-3511. 
[48] T. Kawaguchi, H. Yasuda, K. Shimazu, Electrochemical quartz crystal microbalance investigation of the reductive desorption of self-assembled monolayers of alkanethiols and mercaptoalkanoic acids on Au, Langmuir 16 (2000) 9830-9840.

[49] J.F. Cabrita, L.M. Abrantesa, A.S. Vianaa, N-hydroxysuccinimide-terminated selfassembled monolayers on gold for biomolecules immobilization, Electrochim. Acta 50 (2005) 2117-2124.

[50] A.H. Schmid, S.E. Stanca, M.S. Thakur, K.R. Thampi, C.R. Suri, Site-directed antibody immobilization on gold substrate for surface plasmon resonance sensors. Sens. Actuators B Chem. 113 (2006) 297-303.

[51] K. Won, M.J. Park, H.H. Yoon, J.H. Kim, Immobilization of iron storage protein on a gold electrode based on self-assembled monolayers, Ultramicroscopy 108 (2008), 13421347.

[52] W.E. Rudzinski, K. Francis, Evaluating the surface density and heterogeneity of a dithiobis(succinimidylpropionate) self-assembled monolayer on gold and its coupling with DNA embedded within a matrix, Appl. Surf. Sci. 256 (2010) 5399-5405.

[53] Y. Li, J. Ren, H. Nakajima, B.K. Kim, N. Soh, K. Nakano, T. Imato, Flow sandwich immunoassay for spesific anti-OVA IgG antibody by use of surface plasmon resonance sensor, Talanta 77 (2008) 473-478.

[54] H. Aizawa, Y. Gokita, J.W. Park, Y. Yoshimi, S. Kurosawa, Antibody immobilization on functional monolayers using a quartz crystal microbalance, IEEE Sensors Journal 6 (2006) 1052-1056. 
[55] G. Sakai, S. Nakata, T. Uda, N. Miura, N. Yamazoe, Highly selective and sensitive SPR immunosensor for detection of methamphetamine, Electrochim. Acta 44 (1999) 3849-3854.

[56] G. Sakai, K. Ogata, T. Uda, N. Miura, N. Yamazoe, A surface plasmon resonancebased immunosensor for highly sensitive detection of morphine, Sens. Actuators B Chem. 49 (1998) 5-12.

[57] A. Amine, H. Mohammadi, I. Bourais, G. Palleschi, Enzyme inhibition-based biosensors for food safety and environmental monitoring, Biosens. Bioelectron. 21 (2006) 1405-1423.

[58] D.R. Shankaran, K.V. Gobi, N. Miura, Recent advancements in surface plasmon resonance immunosensors for detection of small molecules of biomedical, food and environmental interest, Sens. Actuators B 121 (2007) 158-177. 


\section{Table of Contents only}

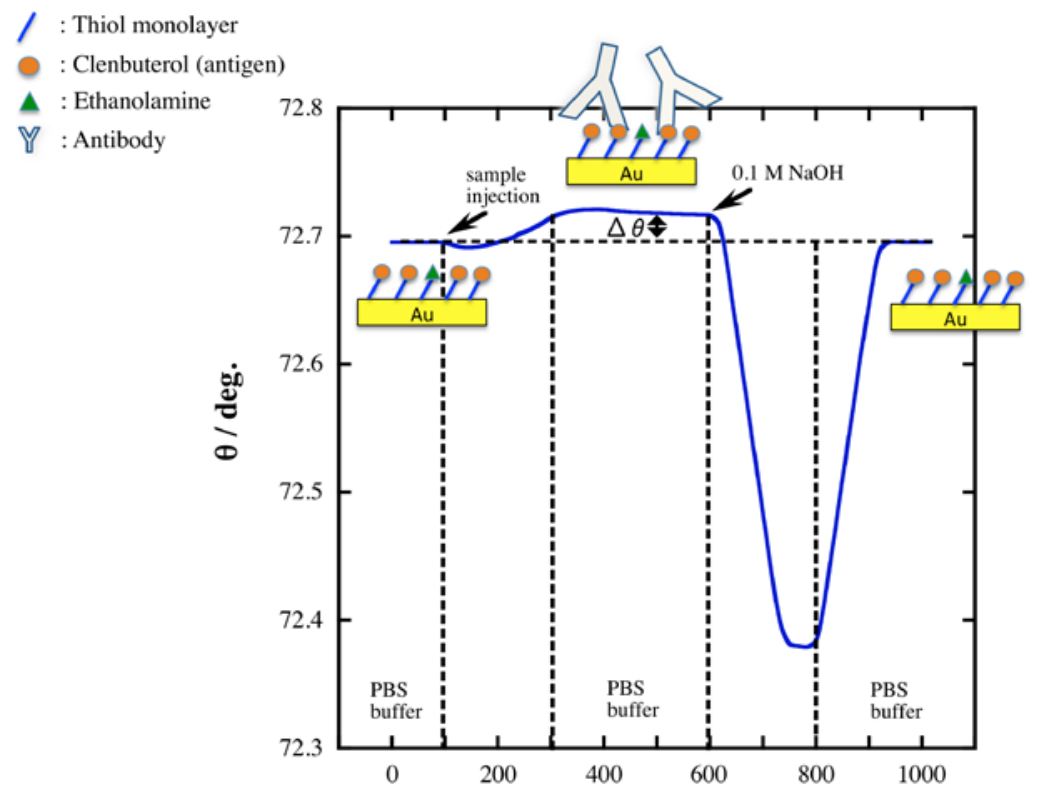

Time / s 


\section{Supporting Information}

Table S1. XPS analysis for DSP on gold surface, in comparison with reductive desorption results.

\begin{tabular}{|c|c|c|c|c|c|c|c|}
\hline \multirow{2}{*}{ DSP on gold surface } & \multicolumn{6}{|c|}{ Atomic composition (\%) } & \multirow{2}{*}{\begin{tabular}{|c|c}
$\begin{array}{c}\text { Reductive } \\
\text { desorption }\end{array}$ \\
$\begin{array}{c}\text { Surface concentration } \\
\left(\times 10^{-10} \mathrm{~mol} \mathrm{~cm}^{-2}\right)\end{array}$
\end{tabular}} \\
\hline & Au $4 \mathrm{f}$ & $\mathrm{Cls}$ & $\mathrm{O} 1 \mathrm{~s}$ & N1s & $\mathbf{S} 2 \mathrm{p}$ & $\mathbf{S} 2 \mathrm{p} / \mathbf{A u} 4 \mathrm{f}$ & \\
\hline $0.1 \mathrm{mM}$ & 37.3 & 31.8 & 22.4 & 2.0 & 6.5 & 17.4 & 5.1 \\
\hline $1.0 \mathrm{mM}$ & 34.6 & 32.2 & 24.5 & 1.9 & 6.8 & 19.6 & 6.1 \\
\hline $5.0 \mathrm{mM}$ & 30.4 & 32.6 & 26.6 & 2.6 & 7.8 & 25.6 & 7.4 \\
\hline $10 \mathrm{mM}$ & 27.8 & 33.4 & 28.6 & 2.5 & 7.7 & 27.7 & 7.7 \\
\hline
\end{tabular}

Table S2. Clenbuterol and ethanolamine coverage on the sensor surface.

\begin{tabular}{|c|c|c|c|c|c|c|}
\hline \multicolumn{2}{|c|}{ Method } & \multirow{2}{*}{$\begin{array}{l}\text { Clenbuterol } \\
9.0 \pm 3.0 \text { mdeg. }\end{array}$} & \multirow{2}{*}{$\begin{array}{c}\text { Ethanolamine } \\
7.1 \pm 2.4 \text { mdeg. }\end{array}$} & \multirow{2}{*}{$\begin{array}{c}\begin{array}{c}\text { Clenbuterol } \\
(\%)\end{array} \\
55.9\end{array}$} & \multirow{2}{*}{$\begin{array}{c}\begin{array}{c}\text { Ethanolamine } \\
(\%)\end{array} \\
44.1\end{array}$} & \multirow{2}{*}{$\begin{array}{l}\text { Assumption } \\
\text { Dielectric }\end{array}$} \\
\hline SPR & $0.1 \mathrm{mM}$ & & & & & \\
\hline & $1.0 \mathrm{mM}$ & $13.4 \pm 2.3$ mdeg. & $8.9 \pm 2.8$ mdeg. & 60.1 & 39.9 & \multirow{3}{*}{$\begin{array}{l}\text { constants/E are } \\
\text { the same as each } \\
\text { solution }\end{array}$} \\
\hline & $5.0 \mathrm{mM}$ & $17.6 \pm 1.7$ mdeg. & $11.3 \pm 2.9$ mdeg. & 60.9 & 39.1 & \\
\hline & $10 \mathrm{mM}$ & $26.2 \pm 5.1$ mdeg. & $13.5 \pm 2.8$ mdeg. & 66.0 & 34.0 & \\
\hline \multirow[t]{4}{*}{ STM } & $0.1 \mathrm{mM}$ & $158.0 \pm 3.4 \mathrm{~nm}^{2}$ & $242.0 \pm 3.4 \mathrm{~nm}^{2}$ & 39.5 & 61.5 & \\
\hline & $1.0 \mathrm{mM}$ & $181.2 \pm 10.7 \mathrm{~nm}^{2}$ & $219.8 \pm 10.7 \mathrm{~nm}^{2}$ & 45.3 & 54.7 & \\
\hline & $5.0 \mathrm{mM}$ & $245.2 \pm 9.8 \mathrm{~nm}^{2}$ & $154.8 \pm 9.8 \mathrm{~nm}^{2}$ & 61.3 & 38.7 & no \\
\hline & $10 \mathrm{mM}$ & $309.6 \pm 8.4 \mathrm{~nm}^{2}$ & $90.4 \pm 8.4 \mathrm{~nm}^{2}$ & 77.4 & 22.6 & \\
\hline \multirow[t]{4}{*}{ XPS } & $0.1 \mathrm{mM}$ & & & 30.5 & 69.5 & Depth of thickness \\
\hline & $1.0 \mathrm{mM}$ & & & 30.5 & 69.5 & coverage are the \\
\hline & $5.0 \mathrm{mM}$ & - & - & 32.2 & 67.8 & same \\
\hline & $10 \mathrm{mM}$ & & & 34.0 & 66.0 & \\
\hline
\end{tabular}

